\title{
TRANSIENT REDUCTION WITHOUT NORMALIZATION OF BRAIN NATRIURETIC PEPTIDE EARLY AFTER HEART TRANSPLANTATION
}

Bernard Geny, MD, PhD, Marguerite Follenius, PhD, Eric Epailly, MD, Arnaud Charpentier, MD,

Gabrielle Brandenberger, PhD, Bernard Eisenmann, MD, Pascal Haberey, MD, and François Piquard, PhD,

Strasbourg, France

Brain natriuretic peptide (BNP), a recently discovered cardiac hormone, is secreted mainly by the cardiac ventricles and has potent diuretic, natriuretic, and vasorelaxant properties. Circulating BNP levels are increased in pa-

From Département de Physiologie, Faculté de Médecine and Service de Chirurgie Cardiovasculaire, Hôpitaux Universitaires, Strasbourg, France.

Received for publication July 3, 1997; accepted for publication Sept. 23, 1997.

Address for reprints: Bernard Geny, MD, PhD, Institut de Physiolgie, Faculté de Médecine, 67085 Strasbourg Cedex, France.

J Thorac Cardiovasc Surg 1998;115:473-5

Copyright (C) 1998 by Mosby, Inc.

$0022-5223 / 98 \$ 5.00+0 \quad \mathbf{1 2 / 5 4} / \mathbf{8 6 3 3 3}$ tients with heart failure in proportion to the severity of the disease and may have important compensatory renal, cardiovascular, and endocrinologic actions. Cardiac transplantation, a recognized treatment for end-stage heart failure, normalizes the neuroendocrine balance, but atrial natriuretic peptide and BNP levels remain elevated in heart-transplant recipients. ${ }^{1}$ Contrasting with the numerous studies focused on atrial natriuretic peptide in heart transplantation $^{2}$ and despite the greater potential beneficial effects of BNP, few data are available concerning the BNP level response to cardiac transplantation. ${ }^{3,4}$

Method and results. Plasma BNP levels were determined by radioimmunoassay with kits from Peninsula Laboratories (Belmont, Calif.) after extraction by Sep Pak C18 cartridges (Waters Chromatography, Milford, Mass.) in 25 patients just before and daily during the first week after either heart transplantation $(n=15)$ or coronary 


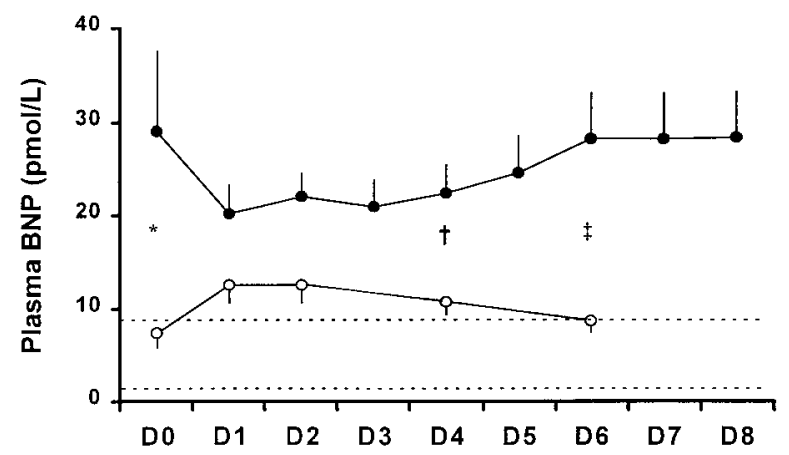

Fig. 1. Plasma BNP concentrations (mean \pm standard error of the mean) before (DO) and during the days (D1 to D8) after heart transplantation $(\mathcal{O}, n=9$ before and $n=$ 15 after) and CABG $(\bigcirc, n=10)$. For differences between patients undergoing heart transplantation and those undergoing CABG, asterisk indicates $p<0.0001$, dagger indicates $p=0.03$, and double dagger indicates $p<0.0001$. The dotted lines show the $95 \%$ range of variation of normal values obtained in healthy subjects.

artery bypass grafting (CABG, $n=10)$. Patients undergoing $\mathrm{CABG}$ served as a control group to differentiate any hormonal effects of cardiac surgery per se from specific effects of transplantation. Cardiovascular hemodynamics, plasma creatinine level (used as an index of renal function), endothelin level, and medications were measured simultaneously. The study was approved by the institutional review board, and each patient gave informed consent.

Fig. 1 shows that plasma BNP level tended to decrease partially and transiently after transplantation, in opposition to the increase observed after CABG. Table I presents the beneficial hemodynamic effects of heart transplantation. CABG transiently increased the heart rate but did not modify the systemic blood pressure; right ventricular hemodynamics were not determined. Serum creatinine level, not modified after CABG, tended to increase after transplantation. As previously reported, endothelin level increased progressively after operation. ${ }^{2}$ Positive correlations were observed between systolic, diastolic, and mean pulmonary artery pressures and BNP level $(r=0.57$, $p=0.01 ; r=0.45, p=0.04$; and $r=0.47, p=0.04$, respectively) and capillary wedge pressure and BNP level ( $r=0.49, p=0.03$ ) when considering the acute effect of transplantation (day 0 and day 1). Among the usual first-week chronotropic and inotropic drug support, only isoproterenol (INN: isoprenaline) and dopamine correlated with plasma BNP level in heart transplantation $(r=$ $0.57, p<0.0001$, and $r=0.23, p<0.02$, respectively). No significant correlation was observed between BNP level and azathioprine, prednisolone, or cyclosporine (INN: ciclosporin), but BNP level was positively correlated with serum creatinine and endothelin levels $(r=0.60, p<$ 0.0001 , and $r=0.55, p=0.002$, respectively).

Discussion. This study confirms that plasma BNP levels are elevated in heart transplantation. It supports, for the first time, the contention that the hemodynamic improve-
Table I. Time course of hemodynamic parameters before and after heart transplantation

\begin{tabular}{lccccc}
\hline & & & & & $p$ \\
& Before & POD I & POD 4 & POD 8 & Value \\
\hline sPAP (mm Hg) & $48 \pm 5$ & $34 \pm 2^{*}$ & $31 \pm 3^{*}$ & - & 0.002 \\
PCWP (mm Hg) & $24 \pm 3$ & $10 \pm 1^{*}$ & $13 \pm 1^{*}$ & - & $<0.0001$ \\
sSBP (mm Hg) & $120 \pm 3$ & $124 \pm 5$ & $133 \pm 5$ & $131 \pm 4$ & 0.06 \\
HR (beats/min) & $81 \pm 4$ & $128 \pm 3^{*}$ & $109 \pm 2^{*}$ & $91 \pm 3$ & $<0.0001$ \\
\hline
\end{tabular}

Data are mean \pm standard error of the mean. Differences between groups were obtained by one-way ANOVA with repeated measures on time. When probability was lower than 0.05 , pairwise comparisons with before value were assessed by Tukey's test. $P O D$, Postoperative day; $s P A P$, systolic pulmonary artery pressure; $P C W P$, pulmonary capillary wedge pressure; $S S B P$, systolic systemic blood pressure; $H R$, heart rate. ${ }^{*} p<0.01$.

ment after cardiac transplantation may partially decrease circulating BNP levels early after the operation. Indeed, we observed positive correlations between BNP level and decreased pulmonary capillary wedge and pulmonary artery pressures in heart transplantation. Accordingly, the right ventricle has been shown to participate in BNP secretion after heart transplantation, ${ }^{3}$ and increased plasma BNP levels were recently reported to be associated with increased right ventricular afterload in heart transplantation. ${ }^{4}$

Several factors acting on BNP clearance and secretion rates may explain why BNP level decreases only partially and transiently after transplantation. BNP is cleared by the kidney, and moderate renal failure, as inferred from increased creatinine level, may partially explain the secondary increase of the cardiac hormone. Endothelin may participate in BNP level elevation in heart transplantation, both through its deleterious renal effects and through direct stimulation of BNP secretion. BNP levels after cardiac transplantation may also reflect predominantly right and left ventricular diastolic function. ${ }^{4}$ Indeed, BNP level is elevated in patients with isolated diastolic dysfunction, ${ }^{5}$ and it is well known that, in addition to normalizing systolic function, heart transplantation results in diastolic dysfunction. This may explain the need for drug support and the positive correlation observed between BNP level and isoproterenol. Although positive correlation between BNP level and trough cyclosporine levels has not consistently been reported, ${ }^{3,4}$ it is tempting to speculate that cyclosporine may participate in BNP level increase in our heart transplantation group, because BNP level increased when cyclosporine therapy was began (day 4). In this view, the lack of correlation between BNP level and cyclosporine in our heart transplantation group may rely on the fact that peak levels or local tissue concentrations of cyclosporine, rather than 12-hour trough level, may be better for cyclosporine effect assessment.

In summary, an early determination of BNP level after transplantation allowed us to observe transient changes that might otherwise have been missed. The results suggest that hemodynamic improvement after heart transplantation reduces plasma BNP levels and that impaired renal function, increased endothelin level, and diastolic dysfunction increase the cardiac hormone early after heart transplantation. Systemic hypertension, occurring later 
after transplantation, may then increase diastolic dysfunction, thus enhancing BNP secretion in heart transplantation.

\section{REFERENCES}

1. Buckley MG, Sethi D, Markandu ND, Sagnella A, Singer DRJ, MacGregor GA. Plasma concentrations and comparisons of brain natriuretic peptide and atrial natriuretic peptide in normal subjects, cardiac transplant recipients and patients with dialysis-independent or dialysis-dependant chronic renal failure. Clin Sci 1992;83:437-44.

2. Geny B, Piquard F, Follénius M, Thiranos JCL, Charpentier A, Epailly E, et al. Endothelin participates in increased circulating atrial natriuretic peptide early after human heart transplantation. J Heart Lung Transplant. In press.

3. Ationu A, Burch M, Singer D, Littleton P, Carter N. Cardiac transplantation affects ventricular expression of brain natriuretic peptide. Cardiovasc Res 1993;27:188-91.

4. El Gamel A, Campbell C, Yonan N, Keevil B, Warburton R, Woodcock A, et al. Atrial natriuretic peptide release after cardiac transplantation. J Thorac Cardiovasc Surg 1996;112: $1128-9$.

5. Lang CC, Prasad N, McAlpine HM, Macleod C, Lipworth BJ, MacDonald TM, et al. Increased plasma levels of brain natriuretic peptide in patients with isolated diastolic dysfunction. Am Heart J 1994;127:1635-6. 